\title{
Prevalence and features of triticea cartilage in an autopsy sample of tertiary care hospital in Sri Lanka
}

Senanayake S.M.H.M.K.

Teaching hospital Anuradhapura

*Corresponding author: Tel: 0094-71-8195569 E-mail address:dilruksena62@yahoo.co.uk

MLJSL. Vol 2. No 1. Mar. pp 1 - 4

\begin{abstract}
Introduction

Triticea cartilage is a grain like cartilage situated just above the tips of the superior thyroid horns. Inexperienced medical officers could mistake this incidental finding for a fracture and thereby an evidence of pressure on the neck.
\end{abstract}

\section{Objective}

This study was designed to find the prevalence of the Triticea cartilage in an autopsy sample in the Kurunegala District of Sri Lanka and if present to ascertain its size and shape.

\section{Methodology}

All autopsies performed by the author during the last four months of the year 2011 were included in to this research.

\section{Results}

The sample studied included 182 males and 67 females $(n=249)$. In males triticea cartilage was present in $101(55.5 \%)$ cases, of which cartilages were present bilaterally in 76 (41.2\%).Unilateral cartilage on the left side was found in $12(6.5 \%)$ of the males and on the right side in $13(7.1 \%)$ cases. Site was just above the tip of the thyroid cartilage in 94 (93\%) cases. Spherical shape of the cartilage was observed in $80(79.3 \%)$ and elongated shape in $21(20.7 \%)$ cases. Triticea cartilage was present in $24(35.8 \%)$ of females of which cartilages were present bilaterally in $14(20.8 \%)$. Unilateral cartilage on the left side was found in $7(10.5 \%)$ of the females and on the right side in $3(4.5 \%)$. Commonest site was just above the tip of thyroid cartilage in $23(95.8 \%)$ cases. Spherical shape in $21(87.5 \%)$ cases and 3 (12.5\%) cases cartilage was elongated.

\section{Conclusion}

This study showed that $50.2 \%$ (half) of the autopsy population has the triticea cartilage. $36.1 \%$ (One third) of population has it on both sides. More than three quarter $(80.8 \%)$ of triticea cartilages are spherical in shape. $92.8 \%$ of triticea cartilages were found just above the tip of superior thyroid horn.

Key words: Triticea cartilage, autopsy sample, presence, prevalence, above the tip of thyroid cartilage, 


\section{Introduction}

Triticea cartilage[1](triticea cartilago), tritiate cartilage[2] or triticious cartilage [3] is a grain like cartilage situated just above the tips of the superior horns of thyroid cartilage [1].

"Tritiate" is a Latin word the meaning of which is grain. Inexperienced medical officers could mistake this incidental finding for a fracture of the thyroid cartilage and conclude that there was evidence of pressure on the neck [4].

Another importance of this cartilage is that fracture of a thyroid horn could be misrecognized as a triticea cartilage. One common postmortem artifact which gained worldwide attention was laryngeal fracture occurring due to handling during the autopsy $[5,6]$. It is possible that much attention was not drawn to the triticea cartilage due to its scarcity in developed countries.

Literature survey showed only a few publications about this cartilage and a few more articles just mentioned this name when dealing with the thyroid cartilage. Even though anatomy text books devote a few sentences to this cartilage, many forensic text books $[7,8]$ do not even mention it. Anatomically it was considered unimportant earlier but now it is accepted as being relevant for strengthening of the thyrohyoid ligament. In the practice of radiology it is important to separate calcified triticea cartilage from a calcified carotid atheroma and soft tissue calcifications [3]. This cartilage has been a frequent finding for the author in the autopsies done in Sri Lanka and was the reason that prompted this research.

\section{Objective}

This study was designed to find the prevalence of the triticea cartilage in autopsies performed in the Kurunegala Teaching Hospital and if present to ascertain its site and shape.

\section{Methodology}

All 249 postmortem examinations (182 males and 67 females) performed by the author during the last four months of the year 2011 were included in to this research. Upper margin of thyroid cartilage is connected by the thyrohyoid membrane to the lower border of the hyoid bone. The thyrohyoid membrane is limited posteriorly by the thyrohyoid ligament. When this ligament is palpated, a grain like nodule is felt somewhere between the tip of the superior thyroid horn and hyoid bone. When soft tissues are dissected away, triticea cartilage is found in the ligament. Rounded and smooth ends help to separate it from a fracture of the thyroid horn because fracture surfaces are flat and irregular [9]. Shape of the cartilage was studied, length was measured and distance between the tip of thyroid horn and triticea cartilage was measured.

\section{Results}

\section{Prevalence}

Out of the 182 males Triticea cartilage was present in 101 (55.5\%) cases. (Table no. 01). Out of these 101 cases, cartilages were present bilaterally in 76 (41.2\%) cases (Table no.02). Unilateral cartilage on the left side was found in $12(6.5 \%)$ cases and on the right side in $13(7.1 \%)$ cases.

In females Triticea cartilage was absent in 43 (64.2\%) cases. Cartilages were present bilaterally in $14(20.8 \%)$ cases. Unilateral cartilage on left side was found in 7 (10.5\%) cases and on the right side in $3(4.5 \%)$ cases.

Table no. 01. Prevalence of cartilage

\begin{tabular}{lllll}
\hline $\begin{array}{l}\text { Presence of } \\
\text { the } \\
\text { cartilage }\end{array}$ & Male & Female & Total & P value \\
\hline Present & 101 & 24 & 125 & 0.005 \\
Absent & 81 & 43 & 124 & \\
Total & 182 & 67 & 249 & \\
\hline
\end{tabular}


Table no. 02.Presence of bilateral and unilateral cartilages-in males and females

\begin{tabular}{lllll}
\hline $\begin{array}{l}\text { Presence of } \\
\text { bilateral cartilage }\end{array}$ & Male & Female & $\begin{array}{l}\text { P } \\
\text { value }\end{array}$ \\
\hline $\begin{array}{l}\text { Bilateral cartilage } \\
\text { present }\end{array}$ & 76 & 14 & 0.103 \\
$\begin{array}{l}\text { Unilateral } \\
\text { cartilage }\end{array}$ & 25 & 10 & \\
\hline
\end{tabular}

\section{Site}

In males, the commonest site was just above the tip of the superior horn of the thyroid cartilage(thyroid horn) in 93(92\%) cases. In 4 cases it was found $0.25 \mathrm{~cm}$ above, in 2 cases $0.5 \mathrm{cmabove}$ and in 2 cases $1 \mathrm{cmabove}$ the tip of the thyroid horn.

In females, commonest site was just above the tip of thyroid horn in $23(95.8 \%)$ cases. In the remaining case cartilage was found $0.5 \mathrm{~cm}$ above the tip of thyroid cartilage.

\section{Shape}

Spherical shape was seen in $80(79.3 \%)$ males and elongated in $21(20.7 \%)$ males. Spherical shape was found in21 (87.5\%) females and elongated in 3 (12.5\%) females.

\section{Size}

Length of the cartilage was variable and measured between 0.25 to one centimeter. Width was equal to the width of its thyroid horn.

\section{Discussion}

This study indicates that $50.2 \%$ of the study population has the triticea cartilage. $36.1 \%$ (slightly more than one third) of the study population has it on both sides. Presence of this cartilage in males is statistically significantly higher than in females. ( $P$ value $=0.005)$.

Presence of bilateral cartilage is statistically significantly higher in males than females. ( $P$ value $=0.103$ ) .
In more than three quarter of the subjects(80.8\%), the cartilage was spherical in shape. In $92.8 \%$ cases it was found just above the tip of the thyroid horn. The size was variable. The length was between $0.25 \mathrm{~cm}$ to one centimeter and the width was equal to the width of the thyroid horn.

Since half the population possess this cartilage, careful neck dissection will reveal it. It is therefore medico legally important to recognize this cartilage and separate it from a fracture of thyroid horn. More than $90 \%$ of the cases it was found just above the tip of the thyroid horn and can be easily mistaken for a fracture of tip of the thyroid horn. In the remaining cases due to the larger gap it is unlikely to be mistaken for a fracture.

One third of the population has it only on one side. When there is a suspicion about neck trauma and if fracture of thyroid horn is found on one side, triticea cartilage on other side may give the misleading impression of bilateral thyroid fractures.

A spherically shaped triticea cartilage can give a false impression of a fracture of the tip of the thyroid horn. An elongated cartilage can give the impression of a fracture of the middle of the thyroid horn. Because of the rounded shape of its lower end, the cartilage can be easily distinguished from a fracture. $X$ ray examination of the larynx will be useful to separate the cartilage from a thyroid fracture, but practically it is not required. A study done on laryngeal cartilages for age changes has not shown any value of calcification of triticea cartilage [10] for assessment of age. On calcification, the triticea cartilage might fuse with the tip of the thyroid horn and give a false impression of an old thyroid fracture [11]. This cartilage is known as a variable cartilage because of embryological developmental variations [12]. This study determined the prevalence, sites, size and shape of Triticea cartilage indicating its importance as one common postmortem artifact found during neck dissections in Sri Lanka. 


\section{Conclusions}

This study showed that $50.2 \%$ (half) of the autopsy population has the triticea cartilage and in $92.8 \%$ the cartilage was found just above the tip of superior thyroid horn. More than one third of the population has it on both sides and more than a three quarter (80.8\%) of the Triticea cartilage were spherical in shape. Because half of the population possess this Triticea cartilage just above the tip of superior thyroid horn, it is a very important, common artifact of neck dissection in Sri Lanka. Awareness of the presence of Triticea cartilage as well as its smooth and rounded upper and lower ends will prevent medical officers from misrecognizing it as a fracture of the tip of the superior horn of the thyroid cartilage.

\section{References}

1. Anderson JE. Grant's Atlas of Anatomy. Williams \& Wilkins 1978; $7^{\text {th }}$ edition.pp-965

2. StandringS.Gray's Anatomy. Elsevier Churchill Livingston; $39^{\text {th }}$ edi.2005 pp-63435

3. Mansur Ahmad et al. Triticeous cartilage: prevalence on panoramic radiographs and diagnostic criteria. Oral Surg Oral Med pathol Oral radiol Endod. 2005;99:225-30.

4. Vanezis P. Pathology of neck injuries. Butterworth. First edition. 1989 p-7

5. Dunsby AM, Davison AM. Causes of laryngeal cartilage and hyoid bone fractures at postmortem. Med Sci Law. 2011 Apr;51(2):109-13

6. Draven KP, Reay DT, Harruff RC. Artifactual injuries of the larynx produced by resuscitative intubation. Am J Forensic Med Pathol. 1999 Mar ;20(1) 31-6

7. Knight B. Forensic pathology. Edward Arnold 1991. $1^{\text {st }}$ edition. pp340-345

8. Mason JK, Purdue BN. The pathology of trauma. Arnold London. $3^{\text {rd }}$ edition.pp241247

9. Charoonnate N, Narongchai P, Vongvaivet S. Fractures of hyoid and thyroid cartilages in suicidal hanging. J Med Assoc Thai. 2010 Oct:93 (10)1211-6

10. Munir Turk L, Hogg DA. Age changes in human laryngeal cartilage. Clinical Anatomy. 1993: 6(3) 154-162

11. Maxeiner $\mathrm{H}$. Healed fractures of the larynx and lingual bone in forensic autopsy. Arch Kriminol. 1999 May-June:203 (5-6): 175-83

12. Grossman JW. Triticeous cartilage. The American Journal of Roentgenology \& Radiotheraphy. Vol L111 no 2 Feb1945. pp 166-170 